\title{
It's the Methodology For Me: A Systematic Review of Early Approaches to Studying TikTok
}

\author{
Shaheen Kanthawala \\ University of Alabama \\ skanthawala@ua.edu
}

\author{
Kelley Cotter \\ The Pennsylvania State \\ University \\ kcotter@psu.edu
}

\author{
Kali Foyle \\ Loyola University \\ Chicago \\ kfoyle@luc.edu
}

\author{
Julia R. DeCook \\ Loyola University \\ Chicago \\ jdecook@luc.edu
}

\begin{abstract}
Research on TikTok has grown along with the app's rapidly rising global popularity. In this systematic review, we investigate 58 articles examining TikTok, its users, and its content. Focusing on articles published in journals and proceedings across the domains of humancomputer interaction, communication, and other related disciplines, we analyze the methods being used to study TikTok, as well as ethical considerations. Based on our analysis, we found that research on TikTok tends to use content analysis as their primary method and mainly focus on user behavior and culture, effects of use, the platform's policies and governance, and very few articles discuss the ethical implications of collecting and analyzing such data. Additionally, most studies employ traditional forms of data collection when the affordances of TikTok tend to differ from other social media platforms. We conclude with a discussion about possible future directions and contribute to ongoing conversations about ethics and social media data.
\end{abstract}

\section{Introduction}

With videos that contain everything from choreographed dances, lip syncs, sea shanties, makeup tutorials, cute animals, political commentary, conspiracy theories, and other memetic content, TikTok's popularity has exploded in recent years and become a strong competitor to other social media giants like Facebook, Twitter. Originating in China as the apps musical.ly in 2014 and Douyin in 2016, the Chinese tech giant ByteDance bought Musical.Ly and folded the app's affordances into the existing Douyin app, creating TikTok and taking the app global in 2018. In just a few short years, TikTok has amassed nearly 2 billion downloads, became the 7th largest social network in the world, and boasted 689 million active users in January 2021 [29].

Although not available in China, when combined with its Chinese counterpart, Douyin, TikTok becomes the 5th largest social network in the world, ahead of Instagram and the Chinese social media platform
WeChat [39]. With the app's continuing growth and unique forms of user content, it has attracted attention from policymakers, academia, and other industry and government sectors. Despite its share of controversy, including Former U.S. President Donald Trump's threat to ban the app and other countries' ban of the app in 2020 [15], the app continues to attract users and grow in its influence.

Research on TikTok is in its nascent stages, and the app has begun to attract more scholarly attention due to its rapidly felt cultural impact, which has resulted in other social media giants cloning the app's functionality (e.g., Instagram's Reels [31]). In this study, we conduct a systematic review to understand how scholars in the fields of human-computer interaction (HCI), communication, and other related disciplines are approaching the app as a venue for academic inquiry. While TikTok shares many similarities with other social media platforms, it is more heavily dependent on algorithmic curation than most other platforms. This difference raises challenges that researchers must respond to by determining the best way to study the platform and its users. Examining the formative research conducted within this space allows us to observe gaps in methodological approaches for this novel platform, opportunities, challenges, and recommend future directions for researchers.

Furthermore, the value of ethical considerations when studying social media data cannot be overlooked and is one that must be grappled with [1]. This research allows us insights into whether/how scholarship tangles with the ethics of user data on a platform predominantly used by younger users (including many minors). Our systematic review focuses on the variety of research undertaken to study the app including methodology, use of human subjects, topical focus, and discussions of the ethical or legal issues of studying TikTok. 


\section{Background}

\subsection{Social Media Research}

Research on TikTok follows a now well-established line of social media inquiry examining both "questions related to social media itself" and "questions that inform our understanding of social phenomena" (McCay-Peet \& Quan-Haase, 2018, p. 19-20). Social media research requires certain methodological considerations particular to the domain. These mainly include questions of representativeness, access, transience, and ethics. In terms of representativeness, past work has demonstrated that social media user populations are skewed in different ways. In general, platforms tend to oversample those of higher socioeconomic status [13]. Different social media also demonstrate differences in gender and age composition--for example, women are more likely to use Facebook, and men are more likely to use Reddit [13]. Further, a small minority of users tend to produce the majority of content circulating on social media [14,27], which creates challenges for sampling. Beyond this, social media trace data are generated by platforms with certain organizational goals in mind, namely enhancing the performance and profitability. These "measurement conditions" shape the insights such data may give rise to as a result of the feedback loop between users and platform governance and design [40].

Related to representativeness, social media platforms do not typically grant unfettered access to data generated by their users. Platforms regulate which data may be accessed and by whom through their APIs and other tools (e.g., Facebook's CrowdTangle). These APIs and tools are subject to constant change, often with little forewarning. Partnerships between platforms and academics have offered one avenue through which researchers can pursue various lines of inquiry [25]. Yet, across APIs, tools, and partnerships, some have expressed concerns about the ways that platforms subtly influence research and scholarship produced from social media data, particularly as platforms supply funding for such work [1,5]. Where, to our knowledge, TikTok has not developed active associations with researchers so far, the challenges arising from the affordances of the platforms also raise issues of representativeness.

Not only can it be difficult to access social media data, but social media platforms evolve iteratively, which makes them unstable targets for research. Social media platforms should be considered "evolving organizations" [11], which exhibit transience, or "change, often dramatically and in short periods of time, in their policies, procedures, and affordances" [4:2]. Such transience often means that even when studies of specific social media platforms offer valuable insights, they represent a particular moment in the history of the platforms. In other words, studies can quickly become outdated as platforms alter features, functionality, and governance structures. The surge in popularity of TikTok as a medium utilized by consumers has led to its rapid growth as a research focus. While TikTok might exhibit transience by evolving, the current interest in the platform, by users and researchers alike, necessitates an examination of how it is being explored.

Social media research also carries novel ethical concerns related to the privacy, anonymity, and consent of human subjects, with differences across researchers in how to approach these [37]. While the public nature of social media content has led many researchers to treat it as "fair game" for data collection and analysis, many scholars have noted that the distinction between public vs. private does not neatly apply to online environments [10]. Instead, social media spaces are characterized by "unsteady forms of publicness" [2]. Private groups and restricted forums are not public, but represent spaces where people might "reasonably expect to be observed by strangers" [35:8], suggesting a more complex sense of privacy. Even when social media data is publicly available, some users expect or prefer that researchers ask for permission before using their content [9].

Finally, many social media users do not have a full and complete understanding of the extent to which their online activity is tracked, which complicates privacy concerns $[10,41]$. Even with such understanding, people may not anticipate that what they post online for particular audiences may be extracted from its original context to be placed under the proverbial microscope [42]. Certainly, gaining consent from social media users for the use of their data presents many practical problems [10]. Although big data collected from social media may be anonymized, without careful oversight, individuals can be easily re-identified if a dataset is made public and/or aggregated with other datasets $[10,41]$. This issue is particularly salient with TikTok where a large volume of users are under the age of 18 .

\subsection{POV: You're a Researcher Interested in Studying TikTok}

TikTok shares various features with other popular social media platforms--for example, the presentation of content in feeds, the ability to "like" and comment on content, and the aggregation of content via hashtags. What sets TikTok apart from most other social media, is how the content on the app is delivered to users and its emphasis on challenges, repeating sounds and visual motifs, and other memetic activity. Whereas most social media platforms heavily depend on users to specify which accounts they wish to see, often centering existing relationships with friends and family members, 
TikTok's main feed (the "For You Page" [FYP]) mainly consists of algorithmically recommended content. This may potentially give the platform more of an authentic or organic feel, as it is likely easier to go viral on TikTok than other platforms where number of followers matters more [22]. Features like the ability to reuse other users' audio and "stitch" a reply to another user's video have rapidly fostered a variety of unique norms and practices on the platform [26] - for example, the oft-cited trend of participating in the latest dance challenge.

These unique affordances make TikTok unlike other social media platforms, and potentially require distinctive methodological approaches of examination. By conducting this systematic review, our goal is to provide an easy to navigate roadmap and reference point for researchers interested in studying TikTok and the emerging cultural phenomena that come from the app, while also noting gaps in scholarship. We additionally aim to contribute to larger conversations in the fields of HCI and communication pertaining to the ethical complexities of studying social media platforms. We are guided by the following research questions:

RQ1: How are scholars exploring TikTok and its users? RQ2: What research methods are scholars using to study TikTok?

RQ3: What challenges and opportunities are there in studying TikTok and its platform culture?

RQ4: What are the ethical challenges in researching TikTok?

\section{Methods}

To begin our systematic review, we first determined the parameters of this work and identified certain inclusion and exclusion criteria outlined below along with our search strategies, coding, and analysis.

\section{Inclusion Criteria}

We narrowed the scope of our study to work in the domains of HCI and communication, targeting relevant sources accordingly (as elaborated below). Beyond this, our primary inclusion criteria was scholarship that substantively studied TikTok or Douyin. In particular, studies that either focused on the platform, such as those examining platform characteristics or those that looked at users, were included. In order for the focus on TikTok to be considered substantive, studies needed to include discussion specific to TikTok (and not just "social media" generally) or needed to use TikTok data in their analysis (e.g. videos and/or other TikTok content).

\section{Search Methods and Exclusion Criteria}

We conducted a search for the terms "TikTok," "Tik Tok," and "Douyin" in relevant databases, namely the Association for Computing Machinery (ACM),
Communication and Mass Media Complete (CMMC), Communication Abstracts, and Web of Science in August 2021. These searches, which were narrowed to scholarly journal articles only, yielded 274 articles in total. This initial search was supplemented with an additional search of the same terms in the top 50 communication journals and top $50 \mathrm{HCI}$ journals identified by SCImago Journal \& Country Rank (https://www.scimagojr.com), which returned another 164 studies.

\section{Selection of Studies}

From the 438 articles found in the aforementioned databases and top journals, 380 were eliminated based on the following criteria:

- The research did not study TikTok, users of TikTok, or did not use any TikTok data

- The study only mentioned TikTok as an example (e.g. "Social media platforms like Instagram and TikTok")

- The study was not in English

- Duplicates studies

After eliminating based on these criteria and general data cleaning, we were left with a total of 58 studies $(n=58)$ for coding. A full flowchart of the study selection process can be found in Figure 1.

\section{Assessment of Methodological Quality/Analysis}

The final sample was coded based on a codebook developed by the authors which focused on methodological approach and the focus of the study. Surveys, interviews, participatory, observational, ethnographic, computational, digital, experimental or quasi-experimental, content analysis and critical or cultural analysis were among the methods by which studies were categorized. For computational approaches, we considered studies that mostly relied on methods such as large-scale analysis (i.e. text and image analysis) whereas digital methods may involve scraping data but use a different analytical framework (e.g. content analysis of scraped data) or techniques like the walkthrough method [20]. We separated participant observation and ethnographic methods from observation because they typically involved interacting with participants, whereas observation does not typically involve direct interaction [3]. These categories were also separated mainly based on the amount of time spent using these methods. Although participant observation is a tool that can be used in ethnography, not all ethnographies use participant observation, and participant observation and general observation can 


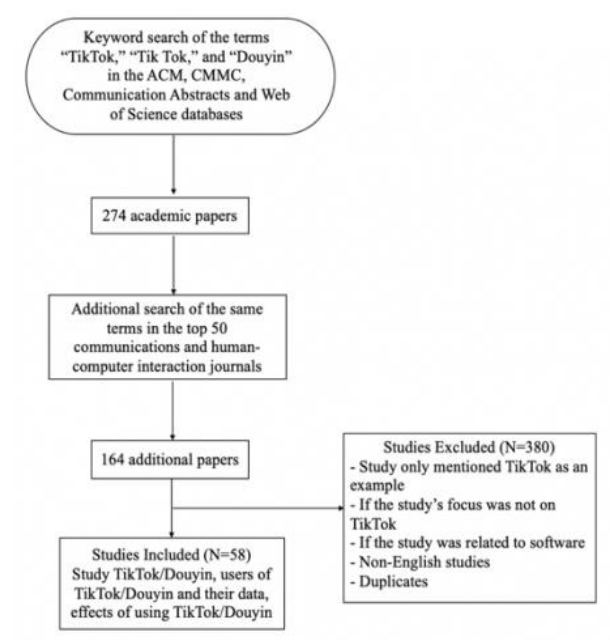

\section{Figure 1. A Flowchart depicting the selection process}

occur in more short-term studies whereas ethnography tends to be more long term [4].

Aside from methodology, studies were coded based on the following variables: primary or secondary research, study focus, age of participants, whether there were human subjects, a focus on TikTok or Douyin; and discussion of ethics. Excepting categories for human subjects and IRB/Ethics discussion, categories were not mutually exclusive, i.e., some studies were coded for multiple dimensions of a single variable (e.g., an article might be coded as focusing on both TikTok and Douyin). We also determined whether a study used primary or secondary research. If data was collected by the authors of a study, it was considered primary; if data was collected for use in a previous study and repurposed in a new one, it was coded as secondary research. Study focus was another area of consideration when analyzing the dataset. The focus of the studies could include the following categories: user behavior; user culture, community and movements; algorithms and software; design and interface; critical analysis or cultural commentary; and media effects/media psychology.

Criterion for the presence of human subjects was also included. Other studies, such as those that performed digital methods research on the platform, but did not include human subjects, were coded accordingly. Particularly for those studies that focused on TikTok users, we noted the age of populations under study based on three groups: youth (12 and under), teens (13-17) and adults (18 and above). Studies that either did not provide distinction of the age of users or did not examine data where age was known or irrelevant, (such as with studying the platform interface itself rather than users), were coded as "not available".

We also distinguished whether the studies focused on TikTok, the global version of the app, or Douyin, the Chinese version of the app, due to slight variations in the characteristics of both platforms. Finally, we coded whether studies discussed ethical considerations related to data collection and/or analysis. A search for keywords, including "ethics" and "Institutional Review Board" was conducted within each manuscript to determine if and how researchers approached ethical considerations. Upon manual review of search results, articles that included any discussion of research ethics were coded accordingly.

\section{Findings}

The first observation within our data was the rapid increase in research focusing on TikTok since 2019, particularly since mid-2020. Figure 2 depicts this interest, indicating the importance of examining the platform and the work being done on it. Based on our analysis, we found that emerging studies on TikTok tend to use content analysis as their primary method and have been mainly interested in the app's user behavior and culture, effects of use, and the platform's policies and governance. All the studies in our sample conducted primary research, with no secondary data analyses. Out of the 58 studies, only 18 studies mentioned either obtaining Institutional Review Board (IRB) or Ethics Board approvals for their studies. Current TikTok research included in this review reflects mainstream concerns about the cultural impact of the app, and conversations about content moderation and the way the platform governs itself are in line with the growing trend of research on platform governance writ large. However, the number of studies that did not address ethics of data collection is concerning, given the app's popularity among minors. A breakdown of the results by category can be found in the table in the supplemental files alongside a numerical list of all the studies used [S1-S58].

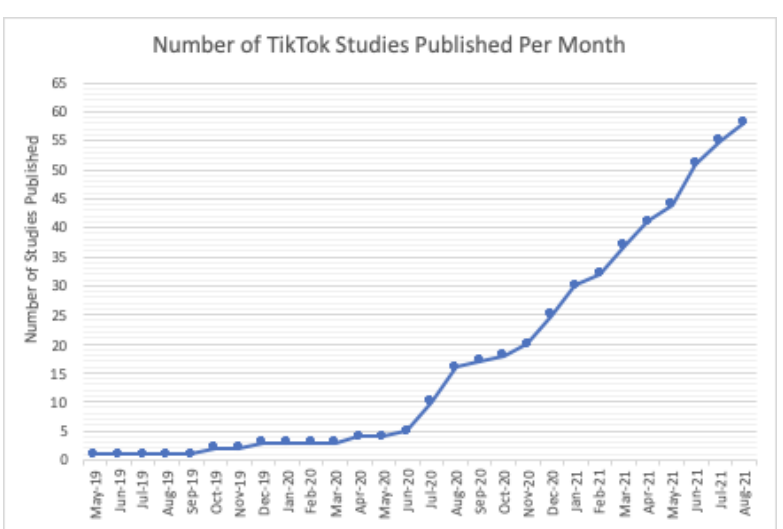

Figure 2. A graph depicting the uptick in TikTok research

\section{Exploring TikTok and its users}


Our first research question asked how scholars were exploring TikTok and its users. Based on our findings, we observed about one-third $(18 / 58)$ of the TikTok studies in our dataset focus on users and their behaviors. This refers specifically to how individual users use the platform and behaviors associated with this use. For instance, Chen, Min, Zhang, Ma, and Evans [S7] examined citizen engagement with government TikTok accounts during the COVID-19 pandemic, and Bucknell Bossen and Kottasz [S6] explored the Uses and Gratifications of using TikTok among young children.

In contrast, close to half of the studies (26/58) focus on user culture. Here, user culture refers to the production of cultural norms and practices on the platform that are not focused on individual users, but instead look at users' collective actions generative of content genres or patterns of behavior on the platform. For instance, Vázquez-Herrero and colleagues [S47] examine how news organizations are adapting to TikTok by tailoring their content to fit TikTok's content style. Additionally, Zulli and Zulli [S58] extended the literature of meme-related research and argue that TikTok encourages imitation and replication (memesis), thus making it a basis of sociality on the app. A few studies $(5 / 58)$ focused specifically on organizational TikTok accounts, indicating an interest in how TikTok is being harnessed for professional and promotional use. Pan and Chi [S36] focused on TikTok accounts of Chinese airlines. Basch, Fera, Pierce and Basch [S2] analyzed videos with the hashtag \#WearAMask alongside videos put forth by the World Health Organization (WHO) in order to explore TikTok's role in community mitigation of the coronavirus.

Less than one-tenth of the studies (4/58) focused on algorithmic and software related research. For instance, both Khoa, Duy, Hoang, Hien and Pham [S18] and Domingues, Nogueira, Francisco and Frade [S9] [7]conducted a forensic analysis of the artifacts left behind by TikTok on Android devices. Meanwhile, Simpson and Seeman [S41] explored how TikTok's FYP algorithm constructs contradictory identity spaces that support and violate the identities of LGBTQ+ users. Ten studies focused on design and interface, which tended to highlight the affordances provided by the platform as well as how features were used. Habibi and Salim [S14] examined TikTok (in addition to Instagram) to see how different types of educational science content led to more user engagement. Meanwhile, Vijay and Gekkar [S48] examined how TikTok's platform design shapes the way politics is performed on it. Seven studies also offered critical analyses or cultural commentaries. For instance, Gray [S13] critically analysed the geopolitics of TikTok through a content analysis of government and company sources and Kaye, Chen and
Zeng [S22] examined the parallel platformization of Douyin and TikTok.

Around one-fifth of the studies (11/58) focused on the effects of TikTok on users individually and as a collective. For instance, Ge, Sui, Zhao and Li [S12] studied the effects of short video ads (on sales. Additionally, in a letter to the editor Ostrovsky and Chen [S35] explained their analysis of COVID-19 information on the platform and how the demand for health content was being surpassed by the supply.

Lastly, a little over one-eighth of the studies (8/58) focused on TikTok's platform or policy governance. For example, Jia and Ruan [S20] compared Chineses apps' data and privacy governance and Weimann and Masri [S50] examined the spread of hate content on TikTok while critiquing the platform for the lack of application of their own Terms of Service to prevent spread of such hate. Out of the 58 studies in our data set, only 27 specified the age of their human subject participants - of these, 17 had adult participants, 7 focused on teens, and only 3 had participants below the age of 12 .

\section{Methods used to explore TikTok}

Our second research question asked about what methods were being employed to study TikTok. As mentioned above, the most commonly employed methodology was content analysis (23/58). These studies largely examined the content of posts on TikTok, most commonly by searching for hashtags or keywords [e.g., S3, S15, S48, S53] and examining top posts returned [e.g., S48] or by or focusing on content from specific kinds of TikTok accounts. These studies examined the post content in detail and contemplated possible impacts.

Digital methods (12/58), critical analyses (9/58), surveys (11/58), and observational studies $(5 / 58)$ were next in frequency. Examples of digital methods utilized included an app walkthrough [e.g., S20, S22, S55] and the use of a web crawler tool [e.g., S7, S15]. Critical analyses covered a range of subjects, for example possibilities for "playful political participation" on TikTok [S48] and how the platform's affordances give rise to affective publics [S15]. All studies that employed surveys focused on users, particularly in consumption rather than production roles. All but one of the observational studies used TikTok as a site for exploring phenomena, analyzing content depicting relevant material. The final observation study consisted of a clinical case report exploring an anorexia patient's use of TikTok. Lastly, there were no more than a couple interviews $(3 / 58)$, focus groups $(1 / 58)$, ethnographies $(3 / 58)$, and experimental studies $(2 / 58)$.

Due to the novel way content on TikTok gets pushed to users and the dissimilar affordances of the platform with regard to potential data collection, we also 
examined if the studies utilized the pre-existing affordances of the platform to assist with data collection. Overall, researchers are not harnessing the pre-existing affordances of the platform to collect data. We do, however, note a few exceptions. For instance, Habibi and Salim [S14] explored static vs. dynamic message delivery for science communication by creating and disseminating posts on TikTok (and Instagram). They measured the "reach" of individual posts, i.e., its views and other engagement by posting the videos to the platform and allowing them to reach their audience through the platform's affordances. This is a novel approach that folds in the algorithmic curation and affordances of the platform, rather than attempting to reach "all users" or a specified sample even, since this is a rough approximation of how content would reach audiences on the platform authentically. Additionally, Schellewald [S38] describes the different forms of communication on TikTok by using the platforms for 30-60 minutes each day for 6 months. Individual affordances of the platform were explored, such as popular sounds and trends. This approach was employed keeping in mind the ephemeral nature of the content on this platform.

Aside from these, most studies (including those that examined the platform's design or affordances as the central focus of the research) utilized traditional methods such as surveys, interviews, or even content analyses, but there largely was no justification for the methodological decisions or explication for why certain methods were appropriate for specific studies.

We also examined the difference in approaches between HCI and communication research. Based on publication outlets, author affiliation, and methodological approach, we determined if each study employed primarily an HCI approach or a communication studies approach. Eleven out of 58 studies were published in predominantly HCI avenues, whereas the remaining fell under the communication studies umbrella using scholarship from communication, journalism, advertising, internet studies, and even health behavior studies. The HCI research (much like communication studies) employed a combination of methods and foci. Three out of the four studies focusing on algorithms came from the HCI field. Overall, the most notable aberrations from past research on major social media platforms $[16,19,30,36,38]$ (as represented in our sample) are relatively more reliance on content analysis and relatively less reliance on surveys and computational approaches.

\section{Challenges and Opportunities}

Based on our findings we identified a number of challenges with studying TikTok and its users as well as a number of opportunities for future work.
While TikTok's algorithm makes the platform uniquely appealing to audiences [33], the algorithm is, in essence, a black box. We note through our findings that only a small number of studies (4/58) target studying TikTok's algorithm and related software. TikTok has endeavored to be transparent about how its algorithm works [21]. In some ways, the platform has been more transparent than other platforms, for example by promising to allow experts to examine its algorithm's source code [23]. Yet, these transparency efforts cannot fully overcome the challenges of studying highly complex algorithms such as TikTok's. The relatively small amount of work exploring TikTok's algorithm could indicate that researchers have not yet formulated effective strategies for such inquiries. Future work in this area might look to past work recommending methodological approaches to studying algorithms "in the wild" [30,31].

Further, while a large volume of our sample were content analyses, wherein researchers collected content from hashtag or search pages, many of these studies did not substantively discuss how algorithmic curation impacts the representativeness of their samples. TikTok's algorithm is heavily dependent on a user's profile (e.g., sign-up information, mobile device information, IP address) and user interactions (e.g., liking, commenting on, or sharing videos) (TikTok, 2019). In past HCI and communication scholarship, researchers have attempted to approximate a ground truth experience by creating fresh user accounts specifically for the purposes of their studies, sometimes using brand new devices [8]. [S32] implemented this strategy in exploring how "Booktok" can be integrated into librarians" reader advisory work "so that the exposure to videos would not be shaped by the researcher's own personal preferences" (p. 3). However, this strategy still creates issues for studies seeking generalizability. For example, as [S39] noted in their study of eating disorder recovery content on TikTok, the algorithm poses challenges for demonstrating the timeliness and relative visibility of certain content on the platform. Relatedly, content analyses like this cannot speak to the relative prevalence of different kinds of content. For example, while [S50] were able to demonstrate the presence of far-right extremist content on TikTok, the authors were careful to avoid quantifications of the magnitude of this presence. As a platform so heavily dependent on algorithmic curation, it is impossible to create a "default" user profile meant to capture an "average" stream of content. This problem is not wholly unique to TikTok. Other social media such as Instagram with its "Top Posts" on its Discover page share this challenge. However, unlike TikTok, Instagram users primarily encounter content via the platform's main feed, which is generated from accounts 
a user follows. Thus, it is easier to estimate exposure to, and perhaps pervasiveness of, content. Researchers interested in studying questions related to TikTok culture, exposure to content, or effects of exposure must take the highly personalized nature of the platform as an inherent limitation. Alternatively, the differences in the platform's affordances can be harnessed for data collection in the future, instead of relying on keyword, hashtag, or top video searches.

To this end however, while some studies in our sample offered useful examples of strategies for identifying specific kinds of users and content on TikTok [e.g., S17, S30, S50], these strategies have limitations related to platform affordances. For example, many studies that targeted specific hashtags for data collection, raising questions about the extent to which those hashtags can stand in for all content addressing a topic or theme. For example, [S30] explained that TikTok limits the number of search results for hashtag queries, but they were unsure of the exact limit, as their multiple searches returned different numbers of results. Moreover, the researchers went on to note that though they attempted to expand their dataset by including "duets"1 with videos returned by their hashtag queries, TikTok does not offer this search functionality. Thus, the researchers instead chose to search by sound, ${ }^{2}$ which TikTok does afford. At a more basic level, researchers' decisions about which keywords, hashtags, or sounds to search for will shape the data they collect in ways that also limit generalizability.

Finally, there are also geographical challenges. TikTok is not accessible in all parts of the world (for instance, the platform is banned in India and used as Douyin in China). Additionally, not all the affordances and filters of the apps are available everywhere. Moreover, as [S17, S53] noted, studies examining English-language hashtags or posts are not representative of TikTok's global user base. Thus, there is no universal TikTok experience, and researchers must take care to acknowledge the variability of use across countries and contextualize findings within the geographical settings of their studies.

Based on our work we can also note a number of opportunities for TikTok research in the future. TikTok is a newer platform as compared to most of the existing social media platforms. Our findings indicate an inherited interest in TikTok policy and governance, extending challenges gleaned from research on other platforms. As the platform has endeavored to be more open about their policies and technologies, TikTok

\footnotetext{
${ }^{1}$ Duets are video responses to other videos on the platform, wherein the "responding" video appears side-by-side with the original video.
}

might prove to be a valuable resource for academic research within this arena.

Additionally, TikTok has a very unique culture, or, more accurately, a unique federation of subcultures. The platform encourages development of norms and memetic replication of content through affordances like the ability to re-use others' audio and "stitch" replies to others' videos, as [S38] demonstrated. This leads to organic community building across not just a usergenerated network, but all users of the platform. This has not only been embraced by users, but actively encouraged by the platform [34]. TikTok's multiplicity and rich well of community connections deserves more attention from both the perspective of content creators as well as consumers.

\section{The Ethics of it All}

Our last research question was about the ethical challenges about studying TikTok and its users. The most significant challenge faced by researchers of the platform is the age of content creators. A very high volume of Tiktok's users are minors [32]. As noted in our results, only 23 studies specified the age of participants, with 17 focusing on adults, 7 on teens and only 3 on people below 12 (a handful of studies had more than one age group e.g., [S6, S42]). This creates a discrepancy in the population that most uses the platform and subset of users being studied. Studying younger populations is challenging, but if we want to understand TikTok, its impact, and its user culture, more research should focus on the most prevalent users of the platform. Related to this, 36 articles in our sample did not use human subjects data, instead relying on data from the platform. Some research did discuss the ethical challenges that go along with this. For example, a few studies noted that they only used publicly available user data, blurred faces in figures, and only collected raw data, such as likes and shares, instead of user profile pictures and handles [S14, S15, S30]. However, this gives rise to two ethical challenges - first, a not insignificant amount of data being collected presumably comes from minors. Although publicly available, special care and consideration should be given to the collection, handling, and publication of such data.

Recognizing the majority of their subjects were minors, one study in our sample [S30] combated this issue by deleting user data after analysis. This is a good measure for ensuring the anonymity of minors, but leaves other challenges, namely that of consent. Fiesler and Proferes [9] showed that few users are knowledgeable about how their content (in this case, tweets) could be utilized for research, and most felt that

\footnotetext{
2 TikTok allows users to make the audio of their videos reusable by other users
} 
this should not be allowed without consent. The authors acknowledged that their findings were dependent on context among other factors, such as who is conducting and disseminating the research and why. In the context of TikTok, one study we reviewed [S17] noted that the terms of service accepted by users when they join the app can be considered consent for collecting user data for research. However, users' awareness and understanding of this point requires additional empirical exploration.

\section{Discussion \& Future Directions}

As an early analysis of the emerging research on TikTok, our systematic review was limited by a relatively small sample, but we believe this systematic review is a good baseline for tracking scholarly interest in the platform over time. In our analysis, we generally observed various common threads from past research on other platforms. For example, like past research on Facebook, Instagram, and Twitter $[6,19,36]$, our sample emphasized general inquiries about how and why people use TikTok. We also noticed some oft-repeated trends in social media research that present unique challenges and opportunities for the exploration of research questions related to TikTok and the evolving social media landscape itself. In recent years, restrictions on social media platforms' APIs, including TikTok's, make it difficult to collect data. In our analysis, the few studies that mentioned scraping data from TikTok did not fully articulate the mechanisms by which they accomplished this. Currently, TikTok's API offers highly limited access to platform data, which has meant a greater emphasis on third-party tools (e.g., [S53] used a web crawler to access publicly available metadata for TikTok videos). Most, however, used more traditional methods such as keyword searches, hashtags, and data collection from the For You page. This points to a lack of a standardized way to collect data from TikTok and study its content. Future research should explore ways to systematically approach this issue, since this problem is not unique to the TikTok platform (e.g. Instagram's "top results" vary from user to user).

TikTok has become a fierce competitor in the social media world, more research on its policy and governance is critically important. For example, as we were preparing this manuscript, news broke that TikTok would start collecting biometric data from its users in the form of faceprints and voiceprints [24], raising further questions about data collection and privacy on the app. Interestingly, while past social media research has emphasized concerns related to information disclosure, privacy, and security [6,39], in our dataset, only two studies [S21, S20] focused on these topics. As another example of the need for more research related to policy and governance, TikTok integrates live content, which is notoriously difficult to moderate and control [12]. In our dataset, surprisingly few articles addressed questions about users' rights or content moderation. Still, this could be more indicative of the pace of academic publishing and scope of our dataset than a shortcoming of extant TikTok research.

In addition, we acknowledge certain limitations of our research. We primarily targeted HCI and communication databases and journals. Certainly, other related fields will have relevant research that we did not assess. TikTok is still a newer platform and research within this space is still on-going. An update to this kind of systematic review would be useful in the future and encouraged to gain a better understanding of how the research has evolved. Another limitation is that we included only studies that were in English, which is only a small portion of the worldwide academic research available. Other valuable research on TikTok has been conducted in other languages, and these works should be included in future research on the topic.

\section{Conclusion}

In this study, we conducted a systematic analysis of research on TikTok in the HCI and communication disciplines to understand how scholarly research is approaching the study of this increasingly popular social media platform. Our results indicated that most research on the app focuses on the emergent culture on the app (i.e. the organic norms and trends by users). Furthermore, researchers also demonstrated some interest in the policy and platform governance of TikTok, an important area with TikTok's promises for future transparency and the high volumes of complex forms of content created (and viewed) by minors.

Methodologically, most of the research employed content analysis, indicating an interest in studying creative output and expression on the platform. However, because of the unique centrality of TikTok's algorithmically curated FYP, the methods used raise questions about how systematic such data collection is and what could be done to standardize such exploration in the future. Finally, from an ethical standpoint, studying TikTok raises multiple challenges, the biggest being its popularity among minors. Because of this, much of the content on the platform is created and viewed by minors. This presents challenges for researchers who must either collect primary data from minors or attempt to exclude this important subset of TikTok users. Further, in studies using publicly available TikTok data, the ethical concerns of whether the content creators are aware of their data being used for research arises. Future work should particularly focus on the ethical challenges faced while studying 
TikTok as a platform, and not conflate it with other social media platforms due to its unique affordances.

\section{References}

[1] Mohamed Abdalla and Moustafa Abdalla. 2021. The Grey Hoodie Project: Big Tobacco, Big Tech, and the threat on academic integrity. arXiv:2009.13676 [cs] (April 2021).

[2] Giovanni Boccia Artieri. 2017. Social Media and the Challenge of Big Data/Deep Data Approach. In Data Science and Social Research. Springer, 57-65.

[3] Paul Atkinson and Martyn Hammersley. 1994. Ethnography and participant observation. In Handbook of Qualitative Research. Sage Publications, Inc, Thousand Oaks, CA, US, 248-261.

[4] Bridget Barrett and Daniel Kreiss. 2019. Platform transience: changes in Facebook's policies, procedures, and affordances in global electoral politics. Internet Policy Review 8, 4 (December 2019). Retrieved June 14, 2021 from https:/policyreview.info/articles/analysis/platformtransience-changes-facebooks-policies-proceduresand-affordances-global

[5] danah boyd and Kate Crawford. 2012. Critical Questions for Big Data. Information, Communication \& Society 15, 5 (June 2012), 662-679.

DOI:https://doi.org/10.1080/1369118X.2012.678878

[6] Ralf Caers, Tim De Feyter, Marijke De Couck, Talia Stough, Claudia Vigna, and Cind Du Bois. 2013. Facebook: A literature review. New Media \& Society 15, 6 (September 2013), 982-1002.

[7] Patricio Domingues, Ruben Nogueira, José Carlos Francisco, and Miguel Frade. 2020. Post-mortem digital forensic artifacts of TikTok Android App. In Proceedings of the 15th International Conference on Availability, Reliability and Security (ARES '20), Association for Computing Machinery, New York, NY, USA, 1-8.

[8] William H. Dutton, Bianca Reisdorf, Elizabeth Dubois, and Grant Blank. 2017. Search and Politics: The Uses and Impacts of Search in Britain, France, Germany, Italy, Poland, Spain, and the United States. Social Science Research Network, Rochester, NY. DOI:https://doi.org/10.2139/ssrn.2960697

[9] Casey Fiesler and Nicholas Proferes. 2018. "Participant" Perceptions of Twitter Research Ethics. Social Media + Society 4, 1 (January 2018).

[10] aline shakti franzke, Anja Bechmann, Michael Zimmer, Charles Ess, and Association of Internet Researchers. 2020. Internet Research: Ethical Guidelines 3.0. Association of Internet Researchers. Retrieved from https://aoir.org/reports/ethics3.pdf

[11] Annabelle Gawer. 2014. Bridging differing perspectives on technological platforms: Toward an integrative framework. Research Policy 43, 7 (September 2014), 1239-1249.

[12] Robert Gorwa, Reuben Binns, and Christian Katzenbach. 2020. Algorithmic content moderation: Technical and political challenges in the automation of platform governance. Big Data \& Society 7, 1 (January 2020), 2053951719897945.

[13] Eszter Hargittai. 2020. Potential Biases in Big Data: Omitted Voices on Social Media. Social Science Computer Review 38, 1 (February 2020), 10-24.

[14] Eszter Hargittai and Gina Walejko. 2008. THE PARTICIPATION DIVIDE: Content creation and sharing in the digital age. Information, Communication \& Society 11, 2 (March 2008), 239256.

DOI:https://doi.org/10.1080/13691180801946150

[15] Jennifer Hassan and Ruby Mellen. 2020. It's not just the United States: These governments also see TikTok as a problem. Washington Post. Retrieved September 4, 2021 from https://www.washingtonpost.com/world/2020/08/03/it s-not-just-united-states-these-governments-see-tiktokgrowing-problem/

[16] Kawaljeet Kaur Kapoor, Kuttimani Tamilmani, Nripendra P. Rana, Pushp Patil, Yogesh K. Dwivedi, and Sridhar Nerur. 2018. Advances in Social Media Research: Past, Present and Future. Inf Syst Front 20, 3 (June 2018), 531-558.

[17] Rob Kitchin. 2017. Thinking critically about and researching algorithms. Information, Communication \& Society 20, 1 (January 2017), 14-29.

[18] Joshua A. Kroll. 2018. The fallacy of inscrutability. Philosophical Transactions of the Royal Society A. Mathematical, Physical and Engineering Sciences 376, 2133 (November 2018).

[19] Linnea Laestadius. 2016. Instagram. In The SAGE Handbook of Social Media Research Methods, Luke Sloan and Anabel Quan-Haase (eds.). SAGE, London, UK, 573-592.

[20] Ben Light, Jean Burgess, and Stefanie Duguay. 2018. The walkthrough method: An approach to the study of apps. New Media \& Society 20, 3 (March 2018), 881900.

[21] Louise Matsakis. 2020. How TikTok's "For You" Algorithm Actually Works. Wired. Retrieved June 13, 2021 from https://www.wired.com/story/tiktokfinally-explains-for-you-algorithm-works

[22] McGlew. 2020. This is How the TikTok Algorithm Works. Later Blog. Retrieved June 15, 2021 from https://later.com/blog/tiktok-algorithm/

[23] Pappas. 2019. TikTok to launch Transparency Center for moderation and data practices. TikTok Newsroom. Retrieved June 14, 2021 from https://newsroom.tiktok.com/en-us/tiktok-to-launchtransparency-center-for-moderation-and-datapractices

[24] Sarah Perez. 2021. TikTok just gave itself permission to collect biometric data on US users, including 'faceprints and voiceprints.' TechCrunch. Retrieved June 14, 2021 from https://social.techcrunch.com/2021/06/03/tiktok-justgave-itself-permission-to-collect-biometric-data-on-us-users-including-faceprints-and-voiceprints/

[25] Cornelius Puschmann. 2019. An end to the wild west of social media research: a response to Axel Bruns. Information, Communication \& Society 22, 11 
(September 2019), 1582-1589.

[26] A Schellewald. 2021. Communicative Forms on TikTok: Perspectives From Digital Ethnography. International Journal of Communication 15, (2021), 1437-1457.

[27] Jen Schradie. 2011. The digital production gap: The digital divide and Web 2.0 collide. Poetics 39, 2 (April 2011), 145-168.

[28] Nick Seaver. 2017. Algorithms as culture: Some tactics for the ethnography of algorithmic systems. Big Data \& Society 4, 2 (December 2017).

[29] Katie Sehl. 2021. 23 Important TikTok Stats Marketers Need to Know in 2021. Hootsuite Blog. Retrieved June 14, 2021 from https://blog.hootsuite.com/tiktok-stats/

[30] Chareen L. Snelson. 2016. Qualitative and Mixed Methods Social Media Research: A Review of the Literature. International Journal of Qualitative Methods 15, 1 (December 2016).

[31] Victoria Song. 2021. Instagram, Honey, Please Stop Copying TikTok. Gizmodo. Retrieved June 14, 2021 from https://gizmodo.com/instagram-honey-pleasestop-copying-tiktok-1846190644

[32] Statista. 2021. U.S. TikTok users by age 2021. Statista. Retrieved June 14, 2021 from https://www.statista.com/statistics/1095186/tiktok-ususers-age/

[33] Joe Tidy and Sophia Smith Galer. 2020. TikTok: The story of a social media giant. $B B C$ News. Retrieved June 14, 2021 from https://www.bbc.com/news/technology-53640724

[34] TikTok. 2021. Subcultures are the new demographics. TikTok for Business. Retrieved June 14, 2021 from https://www.tiktok.com/business/enUS/blog/subcultures-are-the-new-demographics

[35] Leanne Townsend and Claire Wallace. 2016. Social Media Research: A Guide to Ethics. The University of Aberdeen.

[36] Jessica Vitak. 2016. Facebook as a Research Tool in the Social and Computer Sciences. In The SAGE Handbook of Social Media Research Methods, Luke Sloan and Anabel Quan-Haase (eds.). SAGE, London, UK, 627-642.

[37] Jessica Vitak, Katie Shilton, and Zahra Ashktorab. 2016. Beyond the Belmont Principles: Ethical Challenges, Practices, and Beliefs in the Online Data Research Community. Proceedings of the 19th ACM Conference on Computer Supported Cooperative Work and Social Computing (February 2016). DOI:https://doi.org/10.1145/2818048.2820078

[38] Shirley A. Williams, Melissa M. Terras, and Claire Warwick. 2013. What do people study when they study Twitter? Classifying Twitter related academic papers. Journal of Documentation 69, 3 (May 2013), 384-410.

[39] Robert E. Wilson, Samuel D. Gosling, and Lindsay T. Graham. 2012. A Review of Facebook Research in the Social Sciences. Perspect Psychol Sci 7, 3 (May 2012), 203-220.

[40] Angela Xiao Wu and Harsh Taneja. 2020. Platform enclosure of human behavior and its measurement:
Using behavioral trace data against platform episteme. New Media \& Society (July 2020),

[41] Michael Zimmer. 2010. "But the data is already public": on the ethics of research in Facebook. Ethics Inf Technol 12, 4 (December 2010), 313-325.

[42] Michael Zimmer. 2018. Addressing Conceptual Gaps in Big Data Research Ethics: An Application of Contextual Integrity. Social Media + Society 4, 2 (April 2018). 


\section{Appendix A: List of Studies}

\section{LIST OF ARTICLES}

[S1] Avdeeff, M.K., "TikTok, Twitter, and Platform-Specific Technocultural Discourse in Response to Taylor Swift's LGBTQ+ Allyship in 'You Need to Calm Down", Contemporary Music Review, 2021, pp. 1-21.

[S2] Basch, C.H., J. Fera, I. Pierce, and C.E. Basch, "Promoting Mask Use on TikTok: Descriptive, Cross-sectional Study", JMIR Public Health and Surveillance 7(2), 2021, pp. 1-7.

[S3] Basch, C.H., J. Fera, and N. Quinones, "A Content Analysis of Direct-To-Consumer DNA Testing on TikTok", Journal of Community Genetics 12(3), 2021, pp. 489492.

[S4] Basch, C.H., Z. Meleo-Erwin, J. Fera, C. Jaime, and C.E. Basch, "A Global Pandemic in The Time of Viral Memes: COVID-19 Vaccine Misinformation and Disinformation on TikTok", Human Vaccines \& Immunotherapeutics, 2021, pp. 1-5.

[S5] Boatman, D.D., S. Eason, M.E. Conn, and S.K. Kennedy-Rea, "Human Papillomavirus Vaccine Messaging on TikTok: Social Media Content Analysis", Health Promotion Practice, 2021, pp. 1-6.

[S6] Bucknell Bossen, C., and R. Kottasz, "Uses and Gratifications Sought by PreAdolescent and Adolescent Tiktok Consumers", Young Consumers 21(4), 2020, pp. 463-478.

[S7] Chen, Q., C. Min, W. Zhang, X. Ma, and R. Evans, "Factors Driving Citizen Engagement with Government TikTok Accounts During the COVID-19 Pandemic: Model Development and Analysis", Journal of Medical Internet Research 23(2), 2021, pp. 1-13.

[S8] Chen, Z., Q. He, Z. Mao, H.-M. Chung, and S. Maharjan, "A Study on The Characteristics of Douyin Short Videos And Implications For Edge Caching"',

Proceedings of the ACM Turing Celebration Conference - China, ACM (2019), pp. 1-6.

[S9] Domingues, P., R. Nogueira, J.C.

Francisco, and M. Frade, "Post-mortem Digital Forensic Artifacts of TikTok Android App", Proceedings of the 15th International Conference on Availability, Reliability and Security, ACM (2020), pp. 1-8.

[S10] Du, X., T. Liechty, C.A. Santos, and J. Park, "I Want to Record and Share My Wonderful Journey': Chinese Millennials' Production and Sharing of Short-Form Travel Videos on TikTok or Douyin", Current Issues in Tourism, 2020, pp. 1-13.

[S11] Francisco, M.E.Z., and S. Ruhela, "Investigating TikTok as an AI User Platform", 2021 2nd International Conference on Computation, Automation and Knowledge Management (ICCAKM), IEEE (2021), pp. 293-298.

[S12] Ge, J., Y. Sui, X. Zhou, and G. Li, "Effect of Short Video Ads on Sales Through Social Media: The Role of Advertisement Content Generators", International Journal of Advertising 40(6), 2021, pp. 870-896.

[S13] Gray, J.E., "The Geopolitics Of 'Platforms': The TikTok Challenge", Internet Policy Review 10(2), 2021, pp. 1-18.

[S14] Habibi, S.A., and L. Salim, "Static vs. Dynamic Methods of Delivery for Science Communication: A Critical Analysis of User Engagement with Science on Social Media”, PLOS ONE 16(3), 2021, pp. 1-15. 
[S15] Hautea, S., P. Parks, B. Takahashi, and J. Zeng, "Showing They Care (Or Don't): Affective Publics and Ambivalent Climate Activism on TikTok", Social Media + Society 7(2), 2021, pp. 1-14.

[S16] Hayes, C., K. Stott, K.J. Lamb, and G.A. Hurst, "'Making Every Second Count': Utilizing TikTok and Systems Thinking to Facilitate Scientific Public Engagement and Contextualization of Chemistry at Home", Journal of Chemical Education 97(10), 2020, pp. 3858-3866.

[S17] Herrick, S.S.C., L. Hallward, and L.R. Duncan, "'This is Just How I Cope': An Inductive Thematic Analysis Of Eating Disorder Recovery Content Created And Shared on TikTok using \# EDRECOVERY"', International Journal of Eating Disorders 54(4), 2021, pp. 516-526.

[S18] Hoang Khoa, N., P. The Duy, H. Do Hoang, D. Thi Thu Hien, and V.-H. Pham, "Forensic Analysis of TikTok Application to Seek Digital Artifacts on Android Smartphone", 2020 RIVF International Conference on Computing and Communication Technologies (RIVF), IEEE (2020), 1-5.

[S19] Iodice, R., and C. Papapicco, "To be a TikToker in COVID-19 Era: An Experience of Social Influence", Online Journal of Communication and Media Technologies 11(1), 2021, pp. 1-12.

[S20] Jia, L., and L. Ruan, "Going global: Comparing Chinese Mobile Applications' Data and User Privacy Governance at Home and Abroad", Internet Policy Review, 2020, pp. 1-22.

[S21] Kang, H., W. Shin, and J. Huang, "Teens' Privacy Management on VideoSharing Social Media: The Roles of Perceived Privacy Risk and Parental
Mediation", Internet Research ahead-of$\operatorname{print}($ ahead-of-print), 2021, pp. 1-23.

[S22] Kaye, D.B.V., X. Chen, and J. Zeng, "The Co-Evolution of Two Chinese Mobile Short Video Apps: Parallel Platformization of Douyin and TikTok", Mobile Media \& Communication 9(2), 2020, pp. 229-253.

[S23] Klug, D., Y. Qin, M. Evans, and G. Kaufman, "Trick and Please. A MixedMethod Study on User Assumptions about the TikTok Algorithm", 13th ACM Web Science Conference 2021, ACM (2021), pp. 84-92.

[S24] Li, Y., X. Xu, B. Song, and H. He, "Impact of Short Food Videos on the Tourist Destination Image - Take Chengdu as an Example", Sustainability 12(17), 2020, pp. 113.

[S25] Literat, I., "On Research and Hope, in an America Aflame: Sketching Youth Civic Futures as a Mother and a Researcher", Journal of Children and Media 15(1), 2021, pp. 109-111.

[S26] Litzinger, R., and Y. Ni, "Inside the Wuhan Cabin Hospital: Contending Narratives During the COVID-19 Pandemic", China Information, 2021, pp. 1-20.

[S27] Logrieco, G., M.R. Marchili, M. Roversi, and A. Villani, "The Paradox of Tik Tok Anti-Pro-Anorexia Videos: How Social Media Can Promote Non-Suicidal Self-Injury and Anorexia", International Journal of Environmental Research and Public Health 18(3), 2021, pp. 1-4.

[S28] Ma, L., J. Feng, Z. Feng, and L. Wang, "Research on User Loyalty of Short Video App Based on Perceived Value-Take Tik Tok as an Example", 2019 16th International Conference on Service Systems and Service Management (ICSSSM), IEEE (2019), 1-6. 
[S29] Masciantonio, A., D. Bourguignon, P. Bouchat, M. Balty, and B. Rimé, "Don't Put All Social Network Sites in One Basket: Facebook, Instagram, Twitter, TikTok, and Their Relations with Well-Being During the COVID-19 Pandemic", PLOS ONE 16(3), 2021, pp. 1-14.

[S30] Medina Serrano, J.C., O.

Papakyriakopoulos, and S. Hegelich, "Dancing to the Partisan Beat: A First Analysis of Political Communication on TikTok", 12th ACM Conference on Web Science, ACM (2020), pp. 257-266.

[S31] Meng, K.S., and L. Leung, "Factors Influencing TikTok Engagement Behaviors in China: An Examination of Gratifications Sought, Narcissism, and the Big Five Personality Traits", Telecommunications Policy 45(7), 2021, pp. 1-15.

[S32] Merga, M.K., "How Can BookTok on TikTok Inform Readers' Advisory Services for Young People?", Library \& Information Science Research 43(2), 2021, pp. 1-10.

[S33] Ni, Y., M. Fabbri, C. Zhang, and K.A. Stewart, "Reciprocity in Quarantine: Observations from Wuhan's COVID-19 Digital Landscapes", Asian Bioethics Review 12(4), 2020, pp. 435-457.

[S34] Nouwen, M., and M. Hermine Christine Marie Ghislaine D, "TikTok as a Data Gathering Space: The Case of Grandchildren and Grandparents During The COVID-19 pandemic", Interaction Design and Children, ACM (2021), pp. 498-502.

[S35] Ostrovsky, A.M., and J.R. Chen, "TikTok and Its Role in COVID-19 Information Propagation", Journal of Adolescent Health 67(5), 2020, pp. 730.

[S36] Pan, C., and R. Chi, "Analysis and Research on Operation of Tik Tok Accounts of Chinese Airline", 2020 IEEE 2nd International Conference on Civil Aviation Safety and Information Technology (ICCASIT, IEEE (2020), pp. 936-939.

[S37] Rogers, R., "Marginalizing the Mainstream: How Social Media Privilege Political Information", Frontiers in big Data 4, 2021, pp. 1-10.

[S38] Schellewald, A., "Communicative Forms on TikTok: Perspectives from Digital Ethnography", International Journal of Communication; Vol 15 (2021), 202, pp. 1437-1457.

[S39] Scherr, S., and K. Wang, "Explaining the Success of Social Media with Gratification Niches: Motivations Behind Daytime, Nighttime, and Active Use of TikTok in China", Computers in Human Behavior 124, 2021, pp. 1-9.

[S40] Shuhan Gu, J. Ping, M. Xu, and Y. Zhou, "TikTok browsing for anxiety relief in the preoperative period: A randomized clinical trial", Complementary Therapies in Medicine 60, 2021, pp. 1-5.

[S41] Simpson, E., and B. Semaan, "For You, or For 'You'?: Everyday LGBTQ+

Encounters with TikTok", Proceedings of the ACM on Human-Computer Interaction 4(CSCW3), 2021, pp. 1-34.

[S42] Song, S., Y.C. Zhao, X. Yao, Z. Ba, and Q. Zhu, "Short video apps as a Health Information Source: An Investigation of Affordances, User Experience and Users' Intention to Continue the Use of TikTok", Internet Research ahead-of-print(ahead-ofprint), 2021.

[S43] Southwick, L., S.C. Guntuku, E.V. Klinger, E. Seltzer, H.J. McCalpin, and R.M. Merchant, "Characterizing COVID-19 Content Posted to TikTok: Public Sentiment 
and Response During the First Phase of the COVID-19 Pandemic", Journal of Adolescent Health 69(2), 2021, pp. 234-241.

[S44] Su, Y., B.J. Baker, J.P. Doyle, and M. Yan, "Fan Engagement in 15 seconds:

Athletes' Relationship Marketing During a Pandemic via TikTok", International Journal of Sport Communication 13(3), 2020, pp. 436-446.

[S45] Sun, L., H. Zhang, S. Zhang, and J. Luo, "Content-based Analysis of the Cultural Differences between TikTok and Douyin", 2020 IEEE International Conference on Big Data (Big Data), IEEE (2020), pp. 47794786.

[S46] Tang, X., W. Zou, Z. Hu, and L. Tang, "The Recreation of Gender Stereotypes in Male Cross-Dressing Performances on Douyin", Journal of Broadcasting \& Electronic Media, 2021, pp. 1-19.

[S47] Vázquez-Herrero, J., M.-C. NegreiraRey, and X. López-García, "Let's Dance the News! How the News Media are Adapting to the Logic of TikTok", Journalism, 2020, pp. $1-19$.

[S48] Vijay, D., and A. Gekker, "Playing Politics: How Sabarimala Played Out on TikTok", American Behavioral Scientist 65(5), 2021, pp. 712-734.

[S49] Wang, Y., "Humor and Camera View on Mobile Short-Form Video Apps Influence User Experience and Technology-Adoption Intent, an Example of TikTok (DouYin)", Computers in Human Behavior 110, 2020, pp. 1-9.

[S50] Weimann, G., and N. Masri, "Research Note: Spreading Hate on TikTok", Studies in Conflict \& Terrorism, 2020, pp. 1-14.
[S51] Yang, M., S. Hu, B.E. Kpandika, and L. Liu, "Effects of Social Attachment on Social Media Continuous Usage Intention: The Mediating Role of Affective Commitment", Human Systems Management(Preprint), , pp. $1-13$.

[S52] Yu, Z., "An Empirical Study of Consumer Video Activism in China:

Protesting Against Businesses with Short Videos", Chinese Journal of Communication 14(3), 2021, pp. 297-312.

[S53] Zeng, J., and C. Abidin, “"\#OkBoomer, Time To Meet The Zoomers': Studying The Memefication of Intergenerational Politics on TikTok", Information, Communication \& Society, 2021, pp. 1-23.

[S54] Zhang, Z., "Infrastructuralization of Tik Tok: Transformation, Power Relationships, And Platformization of Video Entertainment in China", Media, Culture \& Society 43(2), 2020, pp. 219-236.

[S55] Zhen Ye, "Streamer-Interface-Viewer Entanglement in Popular Chinese Social Media Apps: An Analysis of the Discursive and Affective Live-Streaming Chatroom Interfaces", MedieKultur: Journal of Media \& Communication Research 37(70), 2021, pp. 131-150.

[S56] Zheng, D.X., A.Y. Ning, M.A.

Levoska, L. Xiang, C. Wong, and J.F. Scott, "Acne and Social Media: A Cross-Sectional Study of Content Quality on Tiktok",

Pediatric Dermatology 38(1), 2021, pp. 336338.

[S57] Zhu, C., X. Xu, W. Zhang, J. Chen, and R. Evans, "How Health Communication via Tik Tok Makes a Difference: A Content Analysis of Tik Tok Accounts Run by Chinese Provincial Health Committees", International Journal of Environmental 
Research and Public Health 17(1), 2019, pp. $\quad$ on the TikTok Platform", New Media \& 192.

Society, 2020, pp. 1-19.

[S58] Zulli, D., and D.J. Zulli, "Extending the Internet Meme: Conceptualizing

Technological Mimesis and Imitation Publics 
Appendix B

Results by category

Categories are not mutually exclusive, excepting Human Subjects Research and IRB/Ethics Discussed

\begin{tabular}{|c|c|c|}
\hline Category & Code & Total \\
\hline \multirow{3}{*}{ App Focus } & TikTok & 47 \\
\hline & Douyin & 15 \\
\hline & Both apps & 4 \\
\hline \multirow{2}{*}{ Type of Study } & Primary Research & 58 \\
\hline & Secondary Data Analysis & 0 \\
\hline \multirow{11}{*}{ Methodology } & Survey & 11 \\
\hline & Interviews & 3 \\
\hline & Participatory & 0 \\
\hline & Experimental & 2 \\
\hline & Focus Groups & 1 \\
\hline & Observational & 5 \\
\hline & Ethnographic & 3 \\
\hline & Computational & 5 \\
\hline & Digital Methods & 12 \\
\hline & Content Analysis & 23 \\
\hline & Critical/Cultural Analysis & 9 \\
\hline \multirow{3}{*}{ Age } & Children (12 and under) & 3 \\
\hline & Teens & 7 \\
\hline & Adults & 17 \\
\hline \multirow{6}{*}{ Study Focus } & User Behavior & 18 \\
\hline & User Culture & 26 \\
\hline & Organizations & 5 \\
\hline & Algorithm/Software & 4 \\
\hline & Design and Interface & 10 \\
\hline & Critical Analysis/Cultural Commentary & 9 \\
\hline
\end{tabular}




\begin{tabular}{|c|l|r|}
\hline \multirow{2}{*}{ Human Subjects Research } & Media Effects/Media Psychology & 11 \\
\cline { 2 - 3 } & Platform/Policy Governance & 8 \\
\cline { 2 - 3 } & No & 22 \\
\hline \multirow{2}{*}{ IRB/Ethics Discussed } & Yes & 36 \\
\cline { 2 - 3 } & No & 18 \\
\hline \multirow{3}{*}{ Discipline } & HCI & 40 \\
\cline { 2 - 3 } & Communication & 11 \\
\hline
\end{tabular}

